\title{
Development of a Real-Time ECG Signal Transmission Monitoring Algorithm
}

\author{
Aaron Don M. Africa, Antonio Miguel Sarmiento Alejo, Grant Lewis Milan Bulaong, \\ Samantha Maxine Ronquillo Santos, Jerrick Spencer KehyengUy \\ Department of Electronics and Communications Engineering \\ De La Salle University, Manila \\ 2401 Taft Ave., Malate, Manila 1004, \\ Philippines, aaron.africa@dlsu.edu.ph
}

\begin{abstract}
This research will focus on the creation of a simulated real-time ECG signal transmission Algorithm. A MATLAB program would be created to implement the algorithm and monitor the heart rate of the patient. The study focuses on the implementation of Electrocardiograms (ECG) through simulation and would receive the data through a transmitter to a receiver in real-time. The researchers tackled this topic because of its timeliness in present time society wherein most people neglect their physical health. The researchers will solve this problem through a simulation of an ECG signal using the Matrix Laboratory (MATLAB) software. The system will utilize an ECG sampled signal that was converted digitally so that it could be manipulated by MATLAB. An FSK line encoder will be used, and an additive white Gaussian noise will be applied to simulate real-life noise. The expected results will show that the simulation that will be carried out will create an accurate result with a good signal resolution. This study will prove that the MATLAB software is capable of transmitting and receiving ECG signals.
\end{abstract}

Key words: Electrocardiograms, FSK, Signal Transmissions, Biomedical Systems.

\section{INTRODUCTION}

Signal transmission monitoring increasingly rises in popularity in the medical world [1]. Heart-related diseases, for example, makes use of different technological advances to monitor and test the patients. One monitoring tool which is used in general monitoring of the heart's pace is the electric cardiogram which reads the hearts movement through strategically placed electrodes throughout the body. The signals seen from this device allowed the person in charge like the doctor or the nurse to observe and analyze whether the heart's pace is correct. Additionally, this information could lead to other discoveries related to the patient's health, some example readings found just from an electronic cardiogram reader are the timing of the heartbeat, the pulse, and the heart strength. These machines however still make use of wired connections that restrict some movements for the patient, this restriction could result in a decrease in the comfort of the patient. Technology nowadays, however, introduces different wireless communication systems that enable transmission of data without the restrictions in movement. Wireless communications allow the use of wearable medical devices that enable the monitoring and diagnosis of different parameters found in the body while being able to freely move. These parameters are essential in testing and diagnosis for different types of patients that demand constant supervision and testing. Telemedicine, which takes advantage of wireless communications enable doctor-patient interaction from different locations that could be reached through different forms of communication. While the margin of error for this type of application of wireless communication must be close to none, the challenge for creating a real-time electric cardiogram involves both processing of the data and seamless transmission from one location to another, without any discrepancies to the information being sent. The transmission of the information is to be tackled in this paper, reviewing different types of transmission systems and different communication protocols available.

\section{BACKGROUND OF THE STUDY}

Due to the increasing number of people suffering from health risks, scientists and engineers have worked on creating better technology to counteract these health problems. One of the leading causes of death has been known to be heart-related diseases. These include heart attacks, heart arrhythmias which were caused by abnormal heartbeats, dilated cardiomyopathy, which is caused by weak heart muscles, etc. All of which has led researchers to the development of Telehealth devices [2]. Telehealth was created when medicinal knowledge was combined with telecommunications. The new-found technology has been on the rise as it provided a cost-efficient means to keep track of one's health. This has paved the way for easier management of the patient's records and their heart conditions in real-time as the data would immediately be sent to the system's database [3]. Research has already been made on multi-hopping network systems that utilized a wireless body area network. This utilized sensors that were attached to the human body to seamlessly send data to the receiver. It was stated in studies that this type of network was found to be suitable for implementation in hospitals [4]. The doctors and 
physicians would have access to the data of their patients and could use that information for regular monitoring. The implementation of this network on hospitals allowed the doctors to minimize unnecessary check-ups for already healthy bodies, and their time would be devoted to those who needed to be treated. Additionally, this process would reduce the unnecessary spending on monthly or yearly check-ups because the doctors would be able to monitor the said patient through the network. If any irregularities appeared in the data that was sent by the transceiver, then the doctor would inform the patient to come to the hospital for a check-up or if it were necessary surgery.

\section{STATEMENT OF THE PROBLEM}

Cardiovascular diseases are the cause of 17 million deaths a year [5]. The introduction of the electrocardiogram (ECG) played an important role in the prevention, diagnosis of the abnormality of patients, and rescue of heart disease. The technology has proven useful, saving countless lives since its development. However, there is often a restriction to the access of such technology especially in remote locations [6]. The wireless communication of ECG signals is the key-problem to resolve for in the problem of the remote diagnosis and monitoring of cardiovascular diseases.

\section{SIGNIFICANCE OF THE STUDY}

The study of portable electronic systems has increased due to the development of wireless communication devices, battery-powered biomedical devices, and consumer electronics. These fields of research allow the betterment and the advancement of the medical field; consequently, resulting in more lives being saved per day [7]. One of the main electronic devices commonly used in the hospital environment involved in the capture of the heart pulses and the different movements found in the heart is called the electrocardiogram. This device, which could potentially monitor different illnesses impossible to diagnose with the human eye, allowed hospital personnel to monitor different types of cases found in different patients; some examples include inflammation of the heart, un-synced pulse, increased heart rate, and many more. The study on this device ensures a better, seamless, use of this device in both rural and urban areas. However, this device has still relied on the doctor-patient interaction face to face, this is due to how this machine depends on different types of sensitive sensors that require the patients to be attached to the machine. This mechanic of this device makes this device hard to test different patients at the same time since the electrodes must be attached while keeping the patient still. The proposed real-time ECG monitor, however, enables the doctor-patient interaction to extend the bounds of one visit or estimate on the patient's health; rather enables the observation of the patient while doing different tasks. Also, with the current advancements found in today's society, it could be possible to monitor and diagnose patients without being in the hospital area. This possibility inclined the researchers to investigate the possibility of real-time electrocardiogram signal transmission and what it takes to enable a convenient way to allow a better patient-doctor interaction. Finally, this study would allow faster diagnosis between patient and doctor because of how the real-time ECG signals would be able to reach the doctor ideally instantaneously.

\section{OBJECTIVES OF THE STUDY}

\subsection{General Objective}

To develop an Algorithm that will monitor Real-Time ECG Signal Transmission.

\subsection{Specific Objective}

1) To utilize a MATLAB Simulation model of sampled ECG Signals

2) To transmit the coded data using FSK Transmission

3) To test the variable signal to noise ratio (SNR) of the system.

\section{DESCRIPTION OF THE STUDY}

The proposed system utilizes a MATLAB simulation of a sampled ECG signal with uniformly distributed measurement noise which is then converted in real-time into 8-bit digital data using pulse code modulation (PCM). The coded data was then transmitted using a simulated unipolar line coded FSK scheme. The proposed system also accounts for real-world channel noise via the addition of additive white Gaussian noise (AWGN) of the variable signal-to-noise ratio (SNR). At the receiver, the digital data were reconverted to an analog signal.

\section{METHODOLOGY}

The researchers utilized the DSP System Toolbox of MATLAB which contains an ECG signal simulator. The output of this simulator was configured to have a sampling frequency of $360 \mathrm{~Hz}, 5 \mu \mathrm{V}$ of additive uniformly distributed measurement noise, and a mean heart rate of $70 \mathrm{bpm}$. This signal was sent through a simulated low-pass filter with a cut-off frequency of $100 \mathrm{~Hz}$. Then, the signal was amplified using a multiplication process (equivalent to an instrumentation amplifier). Alternatively, a real ECG signal may be used.

The signal was then pulse-code modulated using 8 bits (256 quantization levels) at a sampling rate of $100 \mathrm{~Hz}$. This resulted in an $800 \mathrm{~Hz}$ digital signal. Finally, the PCM signal was used as the input to a $433 \mathrm{MHz}$ FSK scheme, with an AWGN signal-to-noise ratio of $10 \mathrm{~dB}$, to a standard radio whip antenna. A simulated receiver decodes the output.

\section{REVIEW OF RELATED LITERATURE}

\subsection{Electrocardiograms}


To improve the capability of methods to diagnose, a group of researchers was able to focus on the utilization of high-resolution electrocardiography spectral methods. They applied the electrocardiography spectral methods in terms of analyzing the variability of cardiac cycle spectral analysis results [8]. To implement their work, the researchers proposed a method wherein they initially start with a single dimension analysis of the heart rate variability, similar to that in [9]. That one-dimensional analysis was meant to target the cardiac cycle duration [10]. They then wanted to move onto a multidimensional cardiac parameter analysis. Their multidimensional cardiac parameter analysis was meant to target the location and overall shape or form of the cardiac cycle's peaks.

To prevent, detect, and diagnose cardiovascular diseases at an earlier stage, automatic arrhythmia detection from electrocardiograms have been seen as a pertinent method [11]. More specifically, another method that has proven to be a good solution in terms of simplifying and making signals more immune to noise was the convolutional neural network [12]. The convolutional neural network was seen to be more superior in those categories compared to the traditional and widely commercialized multi-class arrhythmia classification methods. Even so, the convolutional neural network was unable to accept different electrocardiogram signal lengths, did not consider the electrocardiogram's temporal focus well enough, and could barely detect paroxysmal arrhythmias [13]. With all those mentioned problems in terms of the convolutional neural network, the researchers came up with the idea to create a convolutional neural network based on attention and were incremented in time. Their version was seen to be a deep neural network model that could both temporally and spatially fuse information from the electrocardiogram signals through the implementation of the convolutional neural network, attention module, and recurrent cells. In comparison to the regular model, the convolutional neural network of the researchers allowed for more flexible length in terms of input, cut the parameter amount into half, and also reduced computation by more than $90 \%$ in real-time processing. With all the great features, the Attention-based Time-Incremental Convolutional Neural Network allowed all varied length signal processing problems to be exemplified.

With architecture taken from attention modules given, the researchers aimed to make the capability to interpret a convolutional neural network much better and understandable [14]. Their process was tested on an electrocardiogram classification test. Generally, their modules initially created masks capable of selecting a specific set of features to come up with the final prediction. Moreover, through the visualization of those masks, the important decision-making areas of the signal were seen much easier. Their model was trained on their respective logarithmic spectrograms and also their raw signals [15]. Furthermore, in terms of the raw signals, the maps that were generated were not able to perform any significant feature map filtering. On the other hand, in terms of spectrograms, it was easier to see and interpret masks for arrhythmic parts and noise reduction [16].
To effectively treat cardiovascular diseases, electrocardiograph monitoring has proven itself to be one of the best possible methods [17]. Electrocardiographs were specifically able to detect the leading cause of death all over the world, cardiovascular diseases, automatically. The researchers have worked on techniques to extract features and compare their respective performances in Myocardial Infarction, Atrial Fibrillation, and Pericarditis detection $[18,19,20]$. Feature learning has always been one of the major difficulties in classifying electrocardiograms therefore, they split it into linear and nonlinear features, deep learning, and wavelet transform. Their research was carried out with the PhysioNet database to analyze how effective their various feature extraction methods were. Their Pericarditis and Myocardial Infarction classification required information theory-based specialties. Specifically, permutation and approximate entropy, and subband energy were only a few of their remarkable features [21]. Their detection study of the mentioned methods utilizing machine learning displayed promising yields and showed a 92.04\% sensitivity level. In terms of their algorithm for Atrial Fibrillation detection, they utilized statistic, morphology, and spectral features, along with wavelet entropy, which consequently proved itself to be specific by $93 \%$, accurate by $94.1 \%$, and sensitive by $96 \%$ $[22,23]$.

To estimate blood pressure using features taken from electrocardiogram and photoplethysmogram signals, a non-invasive method was created [24,25]. Through machine learning specifically, artificial neural networks, their non-invasive method was meant to estimate blood pressures $[26,27,28]$. Initially, they conducted their experiment on a real data set of more than 2,000 electrocardiogram, blood pressures, and photoplethysmogram signals from patients of various hospitals within the years 2001 up to 2008. More than the usual features, they aimed to utilize a cross-validation method between features to allow for a more robust, accurate, and reliable estimation of the signals and pressures without calibration. Moreover, in comparison to previous works, their results proved to be more accurate in systolic pressure movements. They aimed to improve their results by raising the number of training samples.

\subsection{Signal Transmission}

In all communication systems, the transmission of signals to the receiver from the transmitter has been made possible by digital modulation techniques [29]. The performance of those digital modulation techniques and systems was seen to be the most critical characteristic and a factor behind the success of achieving the best output from the whole communication process[30,31,32]. There were numerous digital modulation schemes, but the most important ones were binary and multilevel carrier digital modulations. Those schemes included amplitude shift keying, phase-shift keying, frequency-shift keying, differential phase-shift keying, quadrature amplitude modulation, quadrature phase-shift keying, differential quadrature phase-shift keying, offset 
quadrature phase-shift keying and system optimization schemes [33,34,35]. Those modulation schemes were important due to their role in the implementation of modern communications systems [36,37]. The researchers analyzed the quadrature phase-shift keying, differential quadrature phase-shift keying, and offset quadrature phase-shift keying principles. They consequently implemented it through the Matrix Laboratory simulations to compare the performance in terms of bit error rate, additive white Gaussian noise, Rayleigh, and Rician fading channels.

\section{THEORETICAL CONSIDERATIONS}

The useful bandwidth of an ECG signal is often less than 100 $\mathrm{Hz}$, but sometimes it can reach up to $1 \mathrm{kHz}$ [38]. It is generally around $1 \mathrm{mV}$ peak-to-peak in the presence of much larger external high-frequency noise, 50 or $60 \mathrm{~Hz}$ interference, and DC electrode offset potential. It is necessary to consider that the transmitter/receiver pair must be capable of communicating at such low frequencies with minimal losses.

Most of the clinically useful information in the ECG is found in the intervals and amplitudes defined by its features (characteristic wave peaks, frequency components, and time duration) [39]. Because of the physiological variability of the QRS complex and various types of noise present in the real ECG signal, it is challenging to accurately detect the QRS complex. The Noise sources that corrupt the raw ECG signals include; Power line interference, muscle noise, and artifacts due to electrode motion [40].

\section{DATA AND RESULTS}

A MATLAB program was utilized to gather the results of the research. Figure 1 shows the analog signal, quantized signal, and the $30 \mathrm{db}$ SNR quantized signal. Figure 2 shows the encoded signal and the demodulated signal with the applied AWGN to account for the noises in real life.

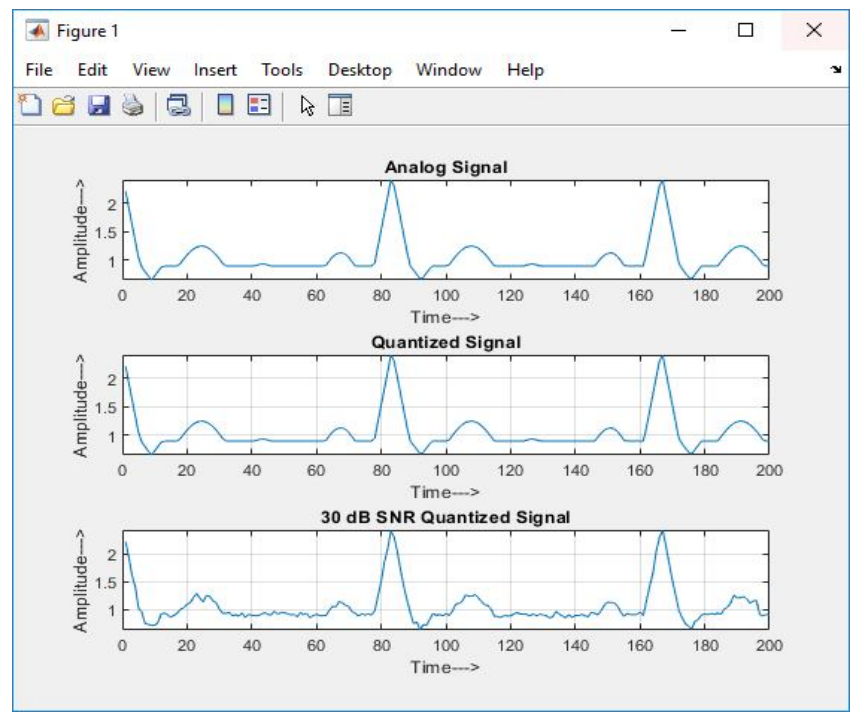

Figure 1: Analog and Quantized Signals

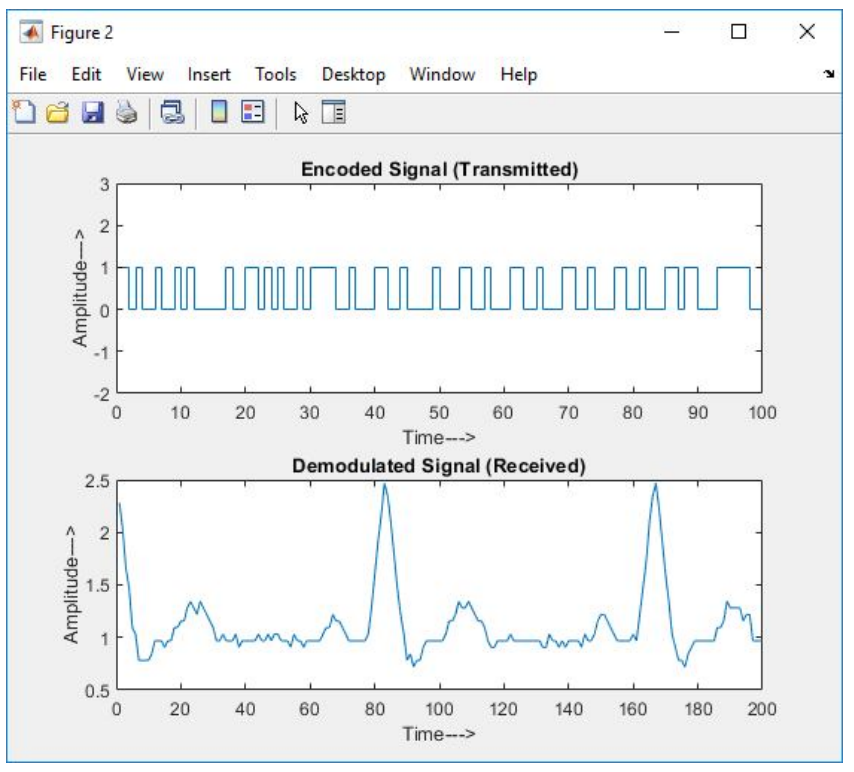

Figure 2: Encoded and Demodulated Signals

\section{ANALYSIS OF DATA}

Because of the use of an FSK modulating scheme, it was found that the proposed system achieves its goal of real-time transmission and reception of ECG signals. Although the MATLAB simulation requires some processing time, the system may be implemented using analog circuits. The acquisition of the ECG signal is often done using proprietary equipment, which introduces no delay. The low-pass filter following the acquisition portion introduces a negligible delay that corresponds to a less than 180-degree phase shift if implemented using RC components. The amplifier may be implemented using operational amplifiers, which introduce negligible delay. The pulse code modulator outputs evenly spaced samples in time. Finally, the FSK receiver and demodulator, when implemented analogically, introduce no delay. The transmission of radio signals through any medium does see some delay due to the distance between the transmitter and receiver; however, this cannot be avoided in most systems.

It was found that the received and demodulated ECG signal may be deemed a faithful reproduction of the transmitted signal. The use of 256 levels of quantization allowed for sufficient signal resolution. Also, PCM sampling the input ECG signal at $100 \mathrm{~Hz}$ resulted in a transmitted digital signal whose bit frequency of $800 \mathrm{~Hz}$ is not rejected by any stray filtering at the receiving end, as well as the elimination of any aliasing due to sampling.

The AWGN that was added in the simulated transmitter channel did not affect the received FSK signal. However, it was found that the simulated additive uniformly distributed measurement noise remained present in the signal. As a future scope, either the transmitter or the receiver may be modified to filter this noise, with the feasibility and practicality of each 
case evaluated. It was clear that the code that was developed in MATLAB is capable of acquiring, transmitting, receiving, and decoding raw ECG signals.

\section{CONCLUSION}

In this research, it was found that with the use of a Frequency-shift keying modulating scheme, the signal that came from the electrocardiogram was able to achieve a real-time transmission and reception. The use of different techniques like low pass filtering the signal as well as phase-shifting the signal introduced delays that would not only cause the simulation to display a shifted output but also display the signal with a cleaner curve, that would follow the original input signal. Besides, the added signal processing steps compensate for the received signals noise interference that would normally be found when operating the ECG machine. Also, the use of an added white Gaussian noise enabled the researchers to test the different parameters used in this project. The quantization level that was chosen was 256 levels, this quantization level allowed the signal resolution to be clear while enabling the signal to be close to the original input signal. The other parameters that were set on this project allowed the receiver to accept the correct signal without incorrect filtering. Hence, one solution found by the researchers to achieve real-time transmission from the ECG machine to the receiver was with the use of analog circuit implementation of FSK receivers in the form of resistive and capacitive components. In conclusion, the project paper was able to achieve a real-time transmission model with the use of different modulating schemes as well as filtering methods that allow fast processing. These signal processing methods, while transformed the input signal to become a cleaner signal, were able to consider the effect of signal transformation, to achieve a fast transition method. Finally, the project design that was presented in this paper was a simulation of what should be reproduced as an analog circuit, to be able to achieve the real-time transmission goal.

\section{RECOMMENDATIONS}

To further improve the study, the researchers suggest that future analysts test different ECG signals. The study only utilized a signal that had uniformly distributed noise. It would have been better if future studies tested if there were noise interferences at different points of the signal. This way, the accuracy of the simulations could be tested and improved. Furthermore, the researchers could also try to obtain an ECG signal that originated from an actual patient. That way, this study could be utilized by those in the medical field due to its usefulness. Another recommendation would be the simplification of the code. Others may be able to find a better way to write the code which would be able to provide a faster and more efficient output. The MATLAB simulation was found to have some delay since the run-time took quite a bit of time to process the data. Creating a better code would reduce this delay and would make the simulation real-time. Moreover, if the researchers would like to carry out the study of the simulation, the creation of a hardware device would be beneficial. They could create a working ECG device that would be able to transmit and receive data from one point to another. Through this implementation, the simulation's results could be corroborated. A comparison study could be done to see if the simulation results would vary from the hardware's results. If there were any notable differences, the simulation must be improved once more. If the data from the hardware and software were found to be similar, then the ECG device could be mass-produced for patients to use to transmit their biomedical data to the doctor in real-time. This could be useful because if there were any irregularities in the patient's ECG, the doctor would be able to see these immediately and could dispatch an ambulance the moment the patient experiences a heart-related problem.

\section{REFERENCES}

[1] B. Babušiak, S. Borik, "Bluetooth Communication for Battery-Powered Medical Devices," Journal of Electrical Engineering. Vol. 67, pp. 65-68, 2013.

[2] D. Simunic, S. Tomac, and I. Vrdoljak, "Wireless ECG Monitoring System," Wireless Communication, Vehicular Technology, Information Theory, and Aerospace \&Electronic Systems Technology. Vol. 1, 2009.

[3] M. Engin, E. Çağlav, and E.Z. Engin, "Real-time ECG Signal Transmission via Telephone Network," Journal of the International Measurement Confederation. Vol. 37, pp. 167-171, 2005.

[4] M.R. Yuce, C.K. Ho, "Implementation of body area networks based on MICS/WMTS medical bands for Healthcare Systems," Personalized Healthcare Through Technology. Vol. 9, pp. 3417-3421, 2008.

[5] Y. Zhu, G. Zhong, "Cardiovascular Disease," Journal of Cardiovascular Disease Research. Vol. 1, No. 1, pp. 3-8, 2010.

[6] J. Pinto, J. Cardoso, \& A. Lourenco, "Evolution, Current Challenges, and Future Possibilities in ECG Biometrics." IEEE Access. Vol. 6, pp. 34746-34776, 2018.

[7] H. Lee, C. Hsu, S. Huang, Y. Shih, and C. Luo, "Designing Low Power Of Sigma-Delta Modulator For Biomedical Application," Biomedical Engineering Applications, Basis, and Communications. Vol. 17, pp. 181-185.

[8] A. Baldin, S. Dosko, K. Kucherov, L. Bin, and A. Spasenov, "ECG Signal Spectral Analysis Approaches for High-Resolution Electrocardiography," Advances in Intelligent Systems and Computing. Vol. 902, pp. 197-209, 2018.

[9] J. Tang, N. Yu, F. Lee, C. Lung, L. Lee, B. Liau, and C. Chen, "Influence of location and frequency variations of binaural electrostimulation on heart rate variability," Advances in Intelligent Systems and Computing. Vol. 967, pp. 70-79, 2019.

[10] H. Ouyang, Z. Liu, N. Li, B. Shi, Y. Zou, F. Xie, Y. Ma, Z. Li, H. Li, Q. Zheng, X. Qu, Y. Fan, and Z. Wang, "Symbiotic cardiac pacemaker," Nature Communications. Vol. 10, No. 1, pp. 281-294, 2019. 
[11] Q. Yao, R. Wang, X. Fan, J. Liu, and Y. Li, "Multi-class Arrhythmia detection from 12-lead varied-length ECG using Attention-based Time-Incremental Convolutional Neural Network," Information Fusion. Vol. 53, pp. 174-182, 2019.

[12] Z. Chen, G. Huang, C. Lu, and G. Chen, "Automatic recognition of weld defects in ToFD D-scan images based on faster R-CNN," Journal of Testing and Evaluation. Vol. 48, No. 2, pp. 696-701, 2019.

[13] R. Rakhmatullov, L. Burmistrova, and I. Moiseeva, "Thyroid status in the combination of atrial fibrillation with subclinical thyrotoxicosis," Bangladesh Journal of Medical Science. Vol. 18, No. 2, pp. 402-410, 2019.

[14] A. Kuvaev, R. Khudorozhkov, "An Attention-Based CNN for ECG Classification," Advances in Intelligent Systems and Computing. Vol. 943, pp. 671-677, 2019.

[15]M. Mulimani, S. Koolagudi, "Acoustic Event Classification Using Spectrogram Features," TENCON. Vol. 10, pp. 1460-1464, 2018.

[16] J. Ruiz, J. Perez, \& J. Blazquez, "Arrhythmia detection using convolutional neural models," Advances in Intelligent Systems and Computing. Vol. 800, pp. 120-127, 2019.

[17] V. Van Toi, "Feature Extraction Techniques for Automatic Detection of Some Specific Cardiovascular Diseases Using ECG: A Review and Evaluation Study," IFMBE Proceedings. Vol. 69, pp. 543-549, 2019.

[18] M. Saqlain, S. Abbas, M. Jae, I. Kang, S. Ali, \& J. Lee, "The Early Prediction Acute Myocardial Infarction in Real-Time Data Using an Ensemble Machine Learning Model," Smart Innovation, Systems and Technologies. Vol. 156, pp. 259-264, 2019.

[19] H. Tien, N. Duc, "Study of the Obstructive Sleep Apnea in the Patients with Cardiovascular Risk," IFMBE Proceedings. Vol. 69, pp. 647-650, 2019.

[20]N. Singh, V. Bhatia, S. Singh, and S. Bhatnagar, "MorCVD: A Unified Database for Host-Pathogen Protein-Protein Interactions of Cardiovascular Diseases Related to Microbes," Scientific Reports. Vol. 9, No. 1, pp. 403-407, 2019.

[21] H. Hosokawa, R. Tamaki, T. Sawada, A. Okonogi, H. Sato, Y. Ogomi, S. Hayase, Y. Okada, and T. Yano, "Solution-processed intermediate-band solar cells with lead sulfide quantum dots and lead halide perovskites," Nature Communications. Vol. 10, No. 1, pp. 808-813, 2019.

[22] A. Arya, B. Bhateja, M. Nigam, and A. Bhadauria, "Enhancement of Brain MR-T1/T2 Images Using Mathematical Morphology," Advances in Intelligent Systems and Computing. Vol. 933, pp. 833-840, 2019.

[23] F. Lilik, L. Solecki, B. Sziova, L. Koczy, and S. Nagy, "On wavelet-based enhancing possibilities of fuzzy classification methods," Advances in Intelligent Systems and Computing. Vol. 945, pp. 56-73, 2019. https://doi.org/10.1007/978-3-030-18058-4_5

[24] N. Maher, G. Elsheikh, W. Anis, and T. Emara, "Non-invasive Calibration-Free Blood Pressure Estimation Based on Artificial Neural Network,"
Advances in Intelligent Systems and Computing. Vol. 921, pp. 701-711, 2019.

[25]P. Yousefian, S. Shin, A. Mousavi, C. Kim, R. Mukkamala, D. Jang, B. Ko, J. Lee, U. Kwon, Y. Kim, and J. Hahn, "The Potential of Wearable Limb Ballistocardiogram in Blood Pressure Monitoring via Pulse Transit Time," Scientific Reports. Vol. 9, No. 1, pp. 107-113, 2019.

[26]P. Galetsi, K. Katsaliaki, and S. Kumar, "Big data analytics in the health sector: Theoretical framework, techniques, and prospects," International Journal of Information Management. Vol. 50, pp. 206-216, 2019.

[27] G. Franchini, P. Burgio, and L. Zanni, "Artificial Neural Networks: The Missing Link Between Curiosity and Accuracy," Advances in Intelligent Systems and Computing. Vol. 941, pp. 1025-1034, 2018.

[28] S.A. Akulove, A.A. Fedotov, I.B. Sidorov, and A.A. Kosheleva, "Influence of artificial Microgravity on Human Arterial Vessels," IFMBE Proceedings. Vol. 73, pp. 655-663, 2019.

[29] H.K. Karim, A.E. Shenger, and A.R. Zerek. "BER Performance Evaluation of Different Phase Shift Keying Modulation Schemes," 19th International Conference on Sciences of Techniques of Automatic Control and Computer Engineering. Vol. 19, pp. 632 -636, 2019.

[30]F. Wu, B.J. Wu, F. Wen, K. andQiu, "All-Optical Modulation Format Conversion from Star-QAM to PSK and ASK Signals," Optics Communications. Vol. 451, pp. 23-27, 2019.

[31] Z. Qian, R. Yan, J. Wu, and X. He, "Full-Duplex High-Speed Simultaneous Communication Technology for Wireless EV Charging," IEEE Transactions on Power Electronics. Vol. 34, pp. 9369-9373, 2019.

[32] P.A. Krishnan, G.K. Jha, and A.B. Walia, "Performance Enhancement of BPSK-SIM- and DPSK-SIM-based FSO Downlink over Atmospheric Turbulence using Aperture Averaging and Receiver Diversity," Photonic Network Communications. Vol. 38, pp. 142 - 150, 2019.

[33] K. Wang, J. Zhang, Y. Li, Y. Zhao, and H. Zhang, "Multi-bit mapping based on constellation rotation in Quantum Noise Stream Cipher,' Optics Communications. Vol. 446, pp. 147-155, 2019.

[34] A. Salleh, M. Aziz, Z. Zakaria, M. Misran, and N. Hashim, "Electromagnetic radiation effect of action potential based on different antenna position in homogeneous human arm flat and cylindrical shape model using ultra wideband coplanar stripline-fed antenna," International Journal of Emerging Trends in Engineering Research (IJETER). Vol. 8, No. 6, pp. 2557-2562, 2020. https://doi.org/10.30534/ijeter/2020/56862020

[35] A. Africa, B. Cajayon, and A. Alano, "Iot technologies systems on medical monitoring and management systems with a real-time ecg signal transmission monitoring algorithm," International Journal of Emerging Trends in Engineering Research (IJETER). Vol. 8, No. 5, pp. 1897-1901, 2020. https://doi.org/10.30534/ijeter/2020/69852020 
[36] S. Malik, P.K. Sahu, "Performance Analysis of Free Space Optical Communication System using Different Modulation Schemes over Weak to Strong Atmospheric Turbulence Channels," Lecture Notes in Electrical Engineering. Vol. 546, pp. 387 - 399, 2019.

[37]D.N. Sankhe, R.R. Swanat, and Y. Srinivas, "Implementation of Harmonic Oscillator using Xilinx System Generator," Advances in Intelligent Systems and Computing. Vol. 941, pp. 503-512, 2018.

[38] S. Mukhopadhyay, S. Mitra, and M. Mitra, "ECG signal compression using ASCII character encoding and transmission via SMS," Biomedical Signal Processing and Control. Vol. 8, No. 4, pp. 354-363, 2013.

[39]B. Biswal, "ECG signal analysis using modified S-transform," Healthcare Technology Letters. Vol. 4, No. 2, pp. 68-72, 2017.

[40] M. Seo, M. Choi, J. Lee, and S. Kim, "Adaptive Noise Reduction Algorithm to Improve R Peak Detection in ECG Measured by Capacitive ECG Sensors," Sensors. Vol. 18, No. 7, pp. 2086, 2018. 